\title{
REAL-TIME COLOR HOLOGRAPHIC VIDEO DISPLAY SYSTEM
}

\author{
Fahri Yaraş, Hoonjong Kang, Levent Onural \\ Bilkent University \\ Department of Electrical and Electronics Engineering \\ TR-06800 Ankara, Turkey \\ fahri@ee.bilkent.edu.tr, hjkang@ee.bilkent.edu.tr, onural@ee.bilkent.edu.tr
}

\begin{abstract}
A real-time multi-GPU color holographic video display system computes holograms from 3D video of a rigid object. System has three main stages; client, server and optics. 3D coordinate and texture information are kept in client and sent online to the server through the network. In the server stage, with the help of the parallel processing ability of the GPUs and segmentation algorithms, phase-holograms are computed in real-time. The graphic card of the server computer drives the SLMs and red, green and blue channels are controlled in parallel. Resultant color holographic video is loaded to the SLMs which are illuminated by expanded light from LEDs. In the optics stage, reconstructed color components are combined by using beam splitters. Reconstructions are captured by a CCD array without any supporting optics. Experimental results are satisfactory.
\end{abstract}

Index Terms - real-time holography, spatial light modulators, phase holograms, three-dimensional displays, computer generated holography

\section{INTRODUCTION}

To achieve a real-time full-color holographic display system is one of the goals of 3DTV applications. However computing fringe patterns and sending them through a data link to the display in real-time are severe bottlenecks. For acceptable video resolutions, holographic fringe patterns may reach up to several megapixels. Time for generating megapixel holographic patterns can take tens of seconds [1]. There are some proposed methods for fast computation of holographic patterns $[2,3,4]$. However they calculate amplitude type holograms, not phase-only holograms. Most of the generation algorithms for phase-only holograms are iterative [5, 6, 7]. We are not aware of any algorithm that generates phase-only holograms in real-time. Accurate Compensated Phase-Added Stereogram (ACPAS) method [8] is used in our system to generate phase-only holograms. They used the term "accurate" to emphasize that reconstructions are quite similar to

This work is supported by EC within FP7 under Grant 216105 with the acronym Real 3D.

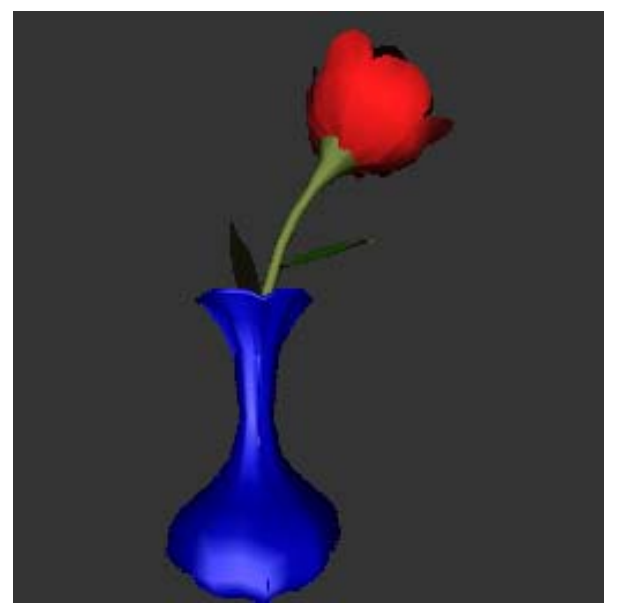

Fig. 1. Rigid 3D Object

the reconstructed images from the Fresnel hologram. ACPAS is slightly lengthy algorithm compared to CPAS [9]; however, the results are comparable to that of Fresnel hologram reconstruction algorithms in terms of quality. We used phaseonly in-line holograms. Phase holograms have some advantageous properties such as low-power diffraction orders, highdiffraction efficiency and low-power undiffracted beam [10, 11]. Together with the useful properties of reflective type phase-only spatial light modulators (SLMs), we benefit from phase holograms [11]. Holographic reconstructions by using LEDs and phase-only spatial light modulators are reported in $[12,13]$.

In coherent optical processing, speckle noise is a severe problem. Many electro-holographic systems use lasers and face with this disturbing phenomenon. There are many methods for reducing the of speckle noise $[14,15,16]$. However they are either more complicated and time consuming or not applicable to holography. Therefore we used LEDs to eliminate those undesired effects. Since LEDs are not hazardous for the eyes, reconstructions can be directly observed by naked eye. Other advantages of LEDs over lasers are ease of operation, being readily available and their low cost. Although LEDs have such desirable properties, quality of holo- 
graphic reconstructions might degrade due to low coherence characteristics. Three different color LEDs are used with three phase-only SLMs to have full color holographic reconstruction $[17,18]$.

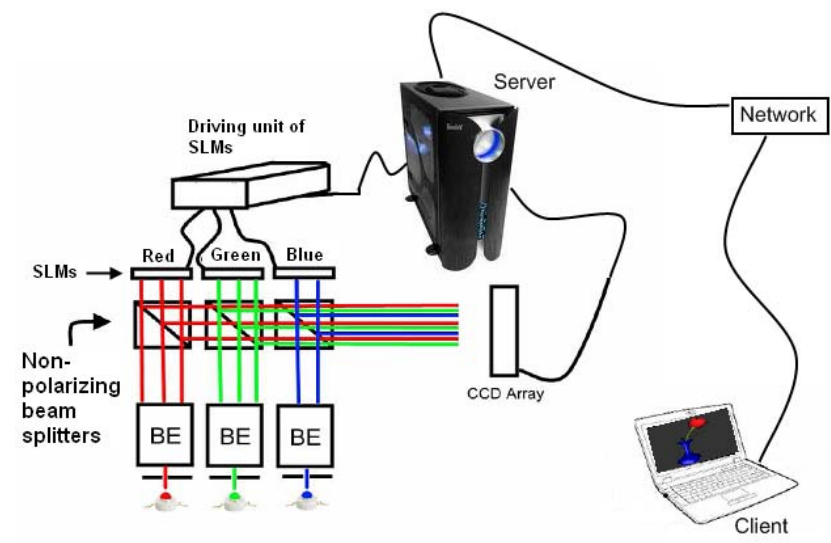

Fig. 2. Overall Setup BE: Beam Expander

A multi-GPU computing architecture is used for real-time color holographic fringe generation. Our 3D model consists of discrete points in space. 3D coordinate information and color value of each point are extracted from each $3 \mathrm{D}$ video frame and send through a network to the server computer. With the help of parallel processing property of the GPU, phase-only holograms are calculated in real-time. Calculated fringe patterns are then sent to the display unit which consists of SLMs, LEDs and optics (Fig.2). The graphic card of the server computer drives the SLMs and red, green and blue channels are controlled in parallel. Received phase-only holograms are loaded to the SLMs and illuminated by corresponding LEDs. Then reconstructed 3D video is captured by the CCD array without any supporting optics.

\section{ALGORITHM}

In our system, we used multi-GPU architecture to calculate real-time holographic video. Flow chart of our proposed method

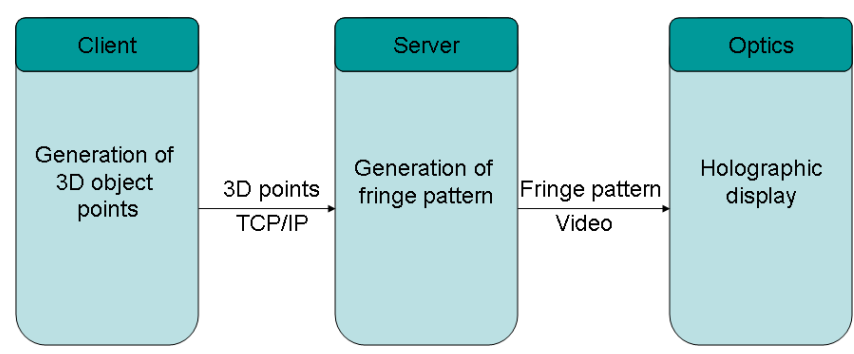

Fig. 3. Flow chart of whole system

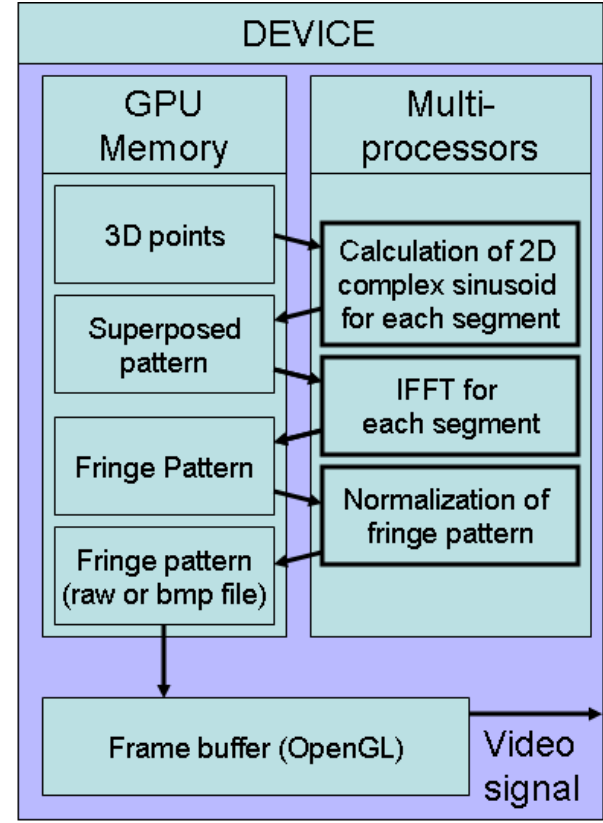

Fig. 4. Flow chart for generating hologram

is shown in Fig.4. 3D video of a rigid 3D object, which consists of points in space, is stored in the client computer (Fig.2). Object rotates in the video. For each frame, RGB color values and the calculated $3 \mathrm{D}$ coordinate information are sent online to server through the network using TCP/IP protocol. The server takes 3D coordinate and texture information from the client for each frame. To use the parallel processing ability of GPU the output diffraction pattern (hologram) partitioned into segments, so that the contribution of each object point approximated as a single 2D complex sinusoid on each segmented hologram pattern. Then for each segment, we superposed the 2D complex sinusoids from all object points. After having a superposed pattern, inverse Fourier transform is taken again in parallel for each segment. Normalization of the resultant fringe pattern completes calculation of the hologram of one 3D video frame. Details of the ACPAS algorithm can be found in [8].

\section{SETUP AND ARCHITECTURE}

There are three main stages in the system; client, server and optics, as shown in Fig.3. 3D coordinates and texture information of each point are kept at the client. At first; for each $3 \mathrm{D}$ video frame, the stored 3D coordinate information and RGB color values are sent online to server through the network using TCP/IP protocol. As described in Sec.2, the server takes 3D coordinate and texture information from client and generate phase hologram of the corresponding 3D frame of the video. The resultant phase hologram has three components; red, green and blue and this forms an RGB image. 
Holograms for three different colors are easily directed to the corresponding SLMs with the help of the graphic card of the server computer. At the last stage of the system, LEDs illuminate those SLMs. As shown in Fig.2, we aligned all three colors with the help of high precision stages to have a color optical reconstruction.

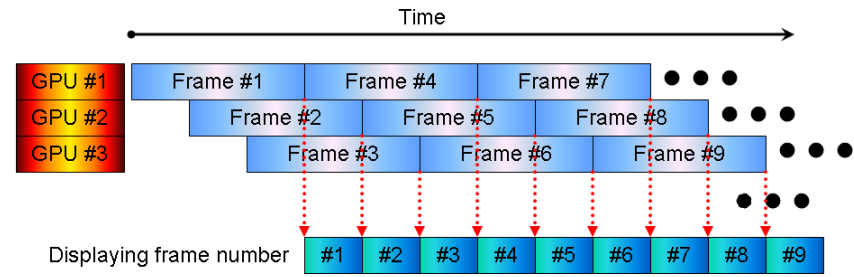

Fig. 5. Frame ordering when three GPUs are used

The server has two Quad Core Intel Xeon E5405 2.0GHz with $12 \mathrm{MB}$ cache processor, 8GB RAM and two Nvidia GTX 280 AMP (512Bit) with 1GB DDR3 GPUs. The client computer has $2 \mathrm{~GB}$ of RAM, $2.0 \mathrm{GHz}$ Intel Core Duo processor. For the optics stage, we used HoloEye's HEO1080P phaseonly spatial light modulators. Number of pixels is $1920 \times 1080$ with $8 \mu m \times 8 \mu m$ square pixel area. Size of the reconstructions is in the order of the SLM size (about $1.5 \times 1.0 \mathrm{~cm}$ ) and the distance of the reconstructions from the SLM is about $60 \mathrm{~cm}$. Dimensions of the each phase hologram is $1920 \times 1080$. Three 1W Edixeon LEDs are used as light sources for red, blue and green whose wavelengths are around $625 \mathrm{~nm}, 460 \mathrm{~nm}$ and $520 \mathrm{~nm}$, respectively. To narrow the spectrum further, the emitted light from LEDs are passed through a pinhole. For uniform planar illumination, beam expanders are used. By using beam splitters reconstructed color components are combined. Reconstructions were taken with a 11 megapixel CCD array without using any supporting optics.

\section{EXPERIMENTAL RESULTS}

In table 1, we have reported the performance analysis of our system. We compare the number of GPUs and the frame rate. We may interpret that the frame rate linearly increases when the number of GPUs increases. The 3D model is shown in Fig.1. Fig.6 illustrates the computer reconstruction using ACPAS algorithm. In Fig.7 optical reconstruction of a single frame of the $3 \mathrm{D}$ video is shown. We used two GPUs to calculate phase holograms for this optical reconstruction.

\begin{tabular}{cccc}
\hline \hline No of Obj. Points & One GPU & Two GPUs & Three GPUs \\
\hline 1 & $15.84 \mathrm{fps}$ & $31.69 \mathrm{fps}$ & $47.53 \mathrm{fps}$ \\
10 & $15.74 \mathrm{fps}$ & $31.48 \mathrm{fps}$ & $47.23 \mathrm{fps}$ \\
100 & $15.38 \mathrm{fps}$ & $30.76 \mathrm{fps}$ & $46.14 \mathrm{fps}$ \\
1000 & $13.51 \mathrm{fps}$ & $27.03 \mathrm{fps}$ & $40.54 \mathrm{fps}$ \\
10000 & $6.84 \mathrm{fps}$ & $13.68 \mathrm{fps}$ & $20.52 \mathrm{fps}$ \\
\hline
\end{tabular}

Table 1. Performance analysis of the system for 2 Megapixel holographic output

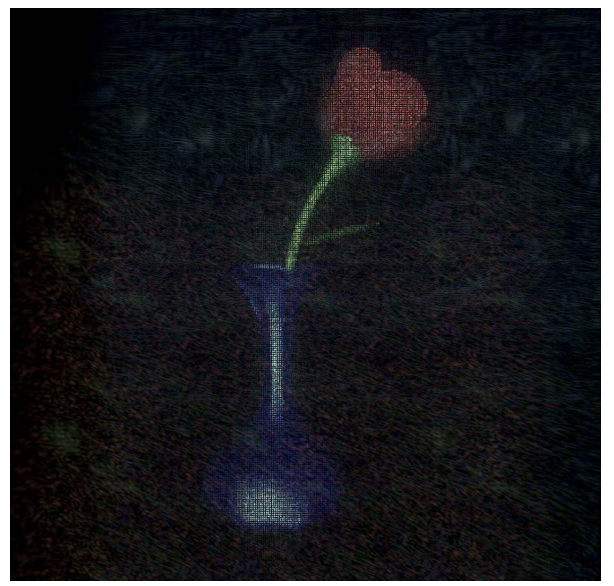

Fig. 6. Computer reconstruction using ACPAS algorithm

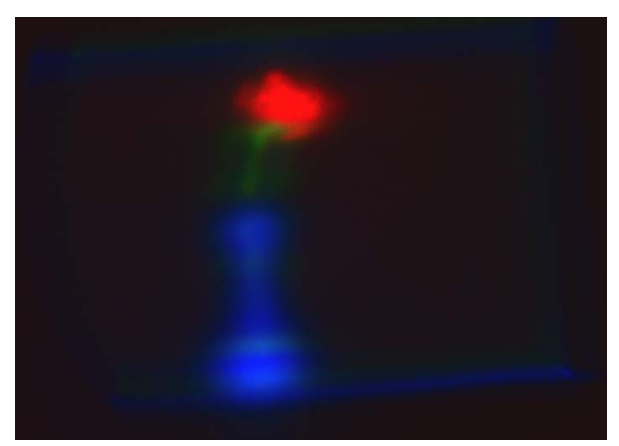

Fig. 7. Optical reconstruction of a single frame of the 3D object. Phase hologram calculated by using two GPUs.

\section{CONCLUSION}

The overall system is integrated successfully and runs as predicted. The optical reconstruction is blurred due to non-coherent light sources and also due to out of focus parts of the reconstructed 3D image. Since the CCD array captures 2D light intensity falling on it, out of focus parts generate blurring. Please note that the image is captured without any supporting optics. Experimental results are satisfactory. It is observed that the proposed system can be used as a color holographic video display. 


\section{REFERENCES}

[1] P.St. Hilaire, S.A. Benton, M. Lucente, Jepsen M.L., J. Kollin, H. Yoshikawa, and J. Underkoffler, "Electronic display system for computational holography," in Proc. SPIE., 1990, vol. 1212, pp. 174-182.

[2] K. Taima, H. Ueda, H. Okamoto, T. Kubota, Y. Nakamura, H. Nishida, H. Takahashi, and E. Shimizu, "New approach to the interactive holographic display system," in Proc. SPIE., 1994, vol. 2176, pp. 23-29.

[3] J.A. Watlington, M. Lucente, C.J. Sparrell, V.M. Bove, and I. Tamitani, "A hardware architecture for rapid generation of electro-holographic fringe patterns," in Proc. SPIE., 1995, vol. 2406, pp. 172-183.

[4] H. Yoshikawa and T. Yamaguchi, "Fast hologram calculation for holographic video display," in Proc. SPIE., 2006, vol. 6027, pp. 561-566.

[5] R. W. Gerchberg and W. O. Saxton, "A practical algorithm for the determination of the phase from image and diffraction plane pictures," Optik, vol. 35, pp. 706-713, 1972.

[6] Alexander V. Kuzmenko and Pavel V. Yezhov, "Iterative algorithms for off-axis double-phase computergenerated holograms implemented with phase-only spatial light modulators," Appl. Opt., vol. 46, no. 30, pp. 7392-7400, 2007.

[7] Chien-Hsien Wu, Chia-Lun Chen, and M. A. Fiddy, "Iterative procedure for improved computer-generatedhologram reconstruction," Appl. Opt., vol. 32, no. 26, pp. 5135-5140, 1993.

[8] H. Kang, "Quality improvements of the coherent holographic stereogram for natural $3 \mathrm{~d}$ display and its applications," in PhD. Thesis, Nihon University, 2008.

[9] H. Kang, T. Fujii, T. Yamaguchi, and H. Yoshikawa, "Compensated phase-added stereogram for real-time holographic display," Opt. Eng., vol. 46, no. 9, pp. 095802, 2007.

[10] L. B. Lesem, P. M. Hirsch, and Jr. J. A. Jordan, "The kinoform: a new wave front reconstruction device," IBM J. Res. Dev., vol. 13, pp. 150155, 1969.

[11] C. Kohler, X. Schwab, and W. Osten, "Optimally tuned spatial light modulators for digital holography," Appl. Opt., vol. 45, no. 5, pp. 960-967, 2006.

[12] F. Yaras, M. Kovachev, R. Ilieva, M. Agour, and L. Onural, "Holographic reconstructions using phase-only spatial light modulators," in 3DTV Conference: The True Vision - Capture, Transmission and Display of $3 D$ Video, 2008, pp. PD-1-PD-4.
[13] M. Kovachev, R. Ilieva, P. Benzie, G. B. Esmer, L. Onural, J. Watson, and T. Reyhan, Holographic 3DTV Displays Using Spatial Light Modulators, Springer, 2008.

[14] Prabakar Puvanathasan and Kostadinka Bizheva, "Speckle noise reduction algorithm for optical coherence tomography based on interval type ii fuzzy set," Opt. Express, vol. 15, no. 24, pp. 15747-15758, 2007.

[15] J. M. Huntley and L. Benckert, "Speckle interferometry: noise reduction by correlation fringe averaging," Appl. Opt., vol. 31, no. 14, pp. 2412-2414, 1992.

[16] Jun Amako, Hirotsuna Miura, and Tomio Sonehara, "Speckle-noise reduction on kinoform reconstruction using a phase-only spatial light modulator," Appl. Opt., vol. 34, no. 17 , pp. 3165-3171, 1995.

[17] F. Yaras and L. Onural, "Color holographic reconstruction using multiple slms and led illumination," in Proc. SPIE., 2009, vol. 7237, p. 723700.

[18] F. Yaras, H Kang, and L. Onural, "Real-time multiple slm color holographic display using multiple gpu acceleration," in Digital Holography and Three-Dimensional Imaging, 2009. 